\title{
Diagnosis of chronic gastric instability using computed tomography in a Great Dane that progressed to gastric dilatation and volvulus: a literature review and case report
}

\author{
Peter Scott Czajkowski* and Ruth Mackenzie Hallman
}

Oklahoma State University, College of Veterinary Health Sciences, 2065 W Farm Rd, Stillwater, OK 74078, USA

\begin{abstract}
Chronic gastric instability (CGI) is part of the gastric dilatation and volvulus (GDV) complex and contributes to the pathophysiologic mechanism of volvulus. Clinical signs can be attributed to a flaccid and mobile stomach. This manuscript reviews the literature regarding CGI and presents a case where computerized tomography is utilized in combination with abdominal radiography to diagnose CGI in an 8 year old spayed female Great Dane. The patient presented for nonspecific clinical signs including hypersalivation, anorexia, and regurgitation, and was euthanized after failing medical management and developing acute abdominal pain. Necropsy revealed a distended stomach with $180^{\circ}$ rotation along its long axis. This case demonstrates that CGI and GDV share a continuum, and cases of CGI may progress to GDV without prophylactic surgical intervention.
\end{abstract}

Keywords: Gastric dilatation and volvulus, Gastric Instability, Gastric Malpositioning, Gastric Torsion.

\section{Introduction}

During radiographic examination of acute gastric torsion in dogs, the stomach often displays a degree of instability, with aboral sections of stomach less fixed in position compared to fundus and cardia (Funkquist, 1969). Frendin et al. (1988) argues that this gastric instability may be present prior to the development of gastric torsion, contributing to the disease. Of less severity but likely similar pathogenesis to gastric dilatation and volvulus (GDV) is chronic gastric instability (CGI). CGI is defined as a flaccidity of the stomach with resulting intermittent gastric malpositioning. There is limited description of CGI in current literature, none of which utilized CT (Boothe and Ackerman, 1976; Matthiesen, 1983; Leib and Blass, 1984; Leib et al., 1987; Frendin et al., 1988; Fossum, 2007; Paris et al., 2011; Sutton et al., 2015). The case presented here is unique in its use of computed tomography to demonstrate gastric instability and thus the dynamic nature of CGI.

\section{Case Details}

An 8-year-old female spayed Great Dane was referred to the Boren Veterinary Teaching Hospital at Oklahoma State University for evaluation of hypersalivation, regurgitation, and anorexia of 10 days duration. Approximately 4 days prior to presentation, the patient was tested for Rocky Mountain Spotted Fever, revealing a 1:512 titer. Oral doxycycline was dispensed and discontinued 2 days later. A series of complete blood counts and biochemistries were performed prior to referral, which revealed a mild thrombocytopenia $(168,000 / \mathrm{uL})$ and mildly elevated
AST (153 units/L), ALT (224 units/L), and ALP (144 units/L). Platelet numbers normalized while being hospitalized at the referring veterinary hospital. Nausea and hypersalivation were treated with a combination of acepromazine, atropine, ginger capsules, diphenhydramine, and maropitant. During hospitalization at the referring veterinary clinic, the patient also began limping on the hind left limb, so dexamethasone IV was started for 2 days.

Upon referral presentation, the patient was lethargic, hyperemic, and dehydrated with slight pain on abdominal palpation noted. Initial database included complete blood count, biochemistry, and urinalysis, which revealed mild hyperglobulinemia $(4.1 \mathrm{~g} / \mathrm{dL})$ as well as mildly elevated ALT (199 IU/L), AST (73 IU/L), ALP (183 IU/L), total bilirubin (0.6 mg/dL), glucose (148 mg/dL), amylase (269 IU/L), and PSL (172 U/L). The CBC revealed a mature neutrophilia $(20,910 / \mathrm{uL})$ with monocytosis $(1476 / \mathrm{uL})$. The urinalysis demonstrated a trace glucosuria, trace ketonuria, 2+ Bilirubinuria, 2+ proteinuria, and alkaline urine $(8.0 \mathrm{pH})$. Maintenance $(60 \mathrm{~mL} / \mathrm{Kg} / \mathrm{d})$ fluid therapy was initiated as well as antiemetic (ondansetron and maropitant), gastroprotectant (pantoprazole), and prokinetic (metoclopramide CRI) therapy.

Thoracic radiography revealed a gas-distended esophagus consistent with megaesophagus as well as a previously diagnosed soft tissue mass in the left caudal lung lobe with tracheobronchial lymphadenopathy. Spondylosis deformans was noted in thoracic vertebrae. Abdominal radiography revealed a non-distended, compartmentalized stomach with a soft tissue band 
separating a smaller gas filled cranially-located portion of the stomach on the right lateral view. On the ventrodorsal view of the abdomen, the stomach was gas filled and was normally positioned. Spondylosis deformans was also noted bridging lumbar vertebrae. Further diagnostics were recommended for both the thoracic and abdominal abnormalities. A complete blood count and biochemistry was repeated 2 days later, which revealed mildly elevated AST (86 IU/L), ALT (141 IU/L), Alk Phos (222 IU/L), total bilirubin (0.4 $\mathrm{mg} / \mathrm{dL})$, and a mature neutrophilia $(17,976 / \mathrm{uL})$ with monocytosis $(1,712 / \mathrm{uL})$. At this time, an acetylcholine receptor antibody test was also performed for myasthenia gravis, which revealed normal titers $(0.14$ mmol/L). Abdominal and thoracic CT were performed. On thoracic CT, a left caudal lobar pulmonary mass was noted (consistent with previously diagnosed mass) as well as multifocal lymphadenopathy and diffuse megaesophagus. An ultrasound-guided aspirate was taken of the pulmonary mass and submitted for cytologic evaluation, which revealed that the mass was a pulmonary carcinoma with suspected intrathoracic lymph node metastasis. Abdominal CT was performed in both sternal and dorsal recumbency (Fig. 1). Multiple hypo-attenuating nodules that did not enhance with contrast were present throughout the hepatic parenchyma as well as an enlarged portal lymph node. The stomach contained a moderate amount of gas and small amount of fluid. On images taken while in sternal recumbency, the pylorus was abnormally positioned to the left of the gastric fundus, with the duodenum running from left to right across the cranial abdomen. When the patient was repositioned in dorsal recumbency, the $\mathrm{CT}$ revealed normal orientation and location of the fundus, pylorus, and proximal duodenum. A diagnosis of CGI without dilatation was made. Recheck abdominal radiographs were performed the next day after the patient became acutely tachycardic and painful on abdominal palpation. On right lateral view, the pylorus was craniodorsally displaced. On dorsoventral views, the stomach appeared compartmentalized. A thin irregular gas opacity that tracked along the ventral and lateral gastric wall, consistent with gastric pneumatosis. These findings were consistent with the gastric dilatation with volvulus. Surgery was recommended, but due to the concurrent intrathoracic disease the owners elected humane euthanasia. Consent was given to perform a post-mortem necropsy. On necropsy, the pulmonary mass was found to be markedly compressing the esophagus, obstructing the lumen. Histopathology of the pulmonary mass confirmed the diagnosis of malignant neoplasm with metastasis to both liver and tracheobronchial lymph nodes, although the precise cell of origin could not be determined on hematoxylin and eosin staining alone.
The stomach was distended, with a $180^{\circ}$ rotation along its long axis. No significant histologic lesions were present in the stomach. The necropsy findings were consistent with previous cytologic and radiographic findings.

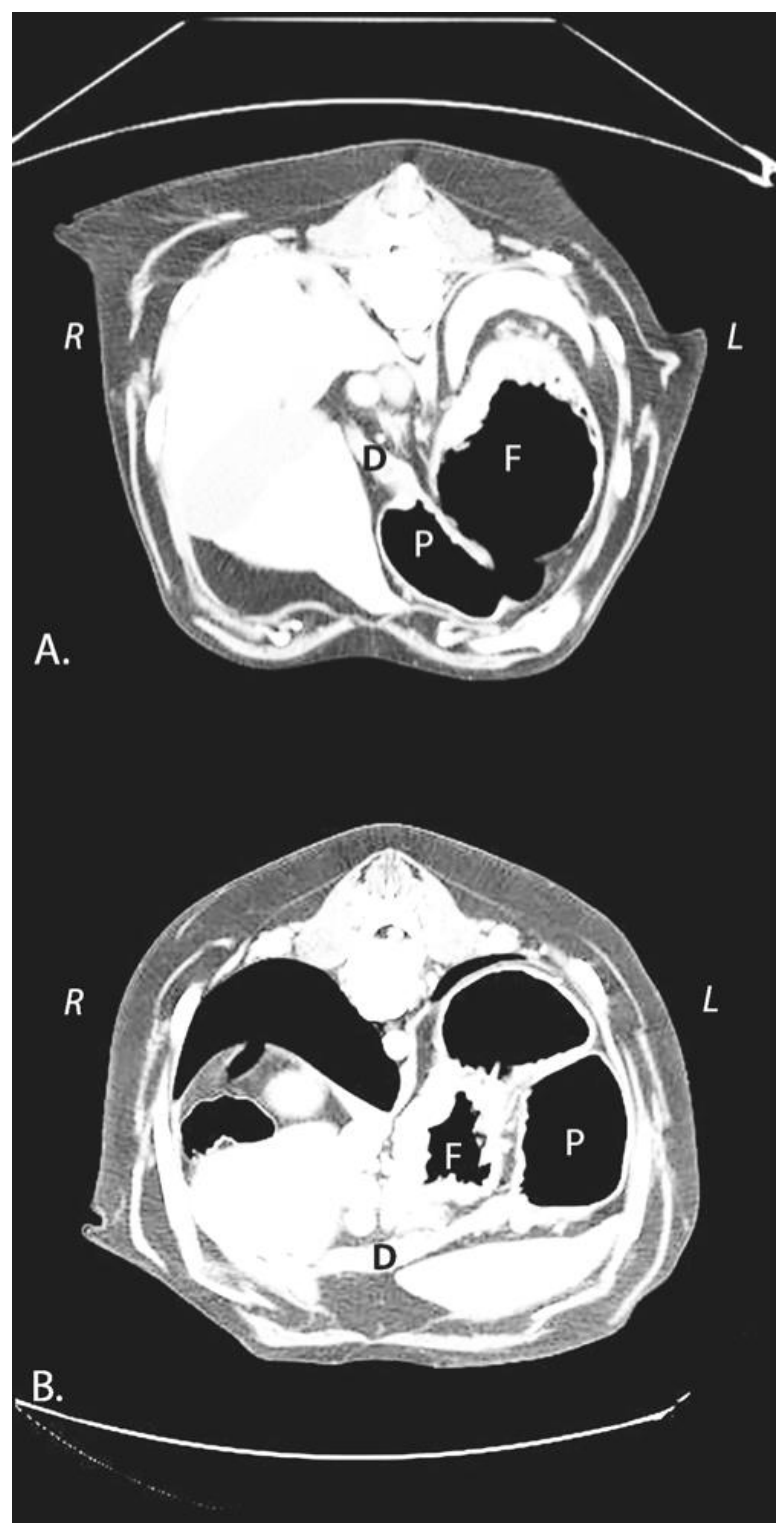

Fig. 1. Computerized Tomography of the presented case demonstrating mobility of the stomach and duodenum. The image is flipped, thus the left side of the patient is on the right side of the images. (F): Fundus; (P): Pylorus; (D): Duodenum. (A): The patient is positioned in dorsal recumbency, but the image has been flipped for easier comparison to Figure 1B. The curved line of the table is visible at the top of the image. The fundus, pylorus, and duodenum are normally positioned. (B): The patient is positioned in sternal recumbency. The pylorus has shifted to the left lateral aspect of the abdomen, and the duodenum runs across the abdomen from left to right. 


\section{Discussion}

GDV is an acute, life-threatening condition that requires immediate medical and surgical intervention (Wingfield et al., 1974; Paris et al., 2011; Gazzola and Nelson, 2014; Sutton et al., 2015). It is typically characterized as a 220-270 degree rotation of the stomach around its mesenteric axis (Fossum, 2007). Mortality rates of acute GDV range from 10-67\% regardless of immediate intensive therapy (Paris et al., 2011).

The etiology of GDV is poorly understood, but purebred large or giant breed dogs has been associated with higher rates of GDV. Glickman et al. (1994) showed that purebred dogs are at a $2.5 \mathrm{x}$ increased risk to develop GDV than mixed breed dogs, and that age significantly influenced susceptibility to GDV. Several large breed dogs, including Great Danes, Gordon Setters, Irish Setters, Weimaraners, Saint Bernards, Standard Poodles, and Basset Hounds are considered predisposed to GDV, suggesting a heritable component to this condition (Tobias and Johnston, 2012; Gazzola and Nelson, 2014).

Other proposed risk factors for the development of GDV include gastric hypomotility, consumption of processed foods, single daily or high-volume feeding, stress, rapidity of ingestion, and high water consumption (Funkquist, 1979; Burrows et al., 1985; Glickman et al., 1994, 1997, 2000a,b; Theyse et al., 1998; Evans and Adams, 2010; Gazzola and Nelson, 2014).

In contrast to GDV, CGI has been described in the literature as partial gastric torsion, partial or chronic GDV, chronic gastric volvulus, gastric malpositioning, and gastric displacement without acute dilatation (Boothe and Ackerman, 1976; Matthiesen, 1983; Leib and Blass, 1984; Leib et al., 1987; Frendin et al., 1988; Fossum, 2007; Paris et al., 2011; Sutton et al., 2015). These manuscripts describe similar presenting signs, mechanisms of diagnosis, and treatment in 17 patients (Boothe and Ackerman, 1976; Matthiesen, 1983; Leib and Blass, 1984; Leib et al., 1987; Frendin et al., 1988; Paris et al., 2011). The most common presenting clinical signs include acute to intermittent vomiting, anorexia, chronic weight loss, and intermittent abdominal bloating. One report describes a case where signs consistent with CGI were the result of a prophylactic gastropexy (Sutton et al., 2015).

Diagnostic plans varied slightly among these reported cases, but each utilized abdominal radiography. In the cases where gastric positioning was abnormal on standard abdominal radiographs, the description of the stomach positioning varied from craniodorsal displacement of the pylorus on lateral view to displaced cardia to the right of midline on ventrodorsal views.
Other cases reported fundus displacement to the left of midline.

Ten of the 17 cases describe utilization of barium contrast to more clearly identify gastric landmarks on radiographs. Fluoroscopy was included in 4 cases. Abdominal ultrasound was utilized in 3 cases, and 4 separate cases also employed upper gastrointestinal endoscopy. Biopsy samples of 5 cases were taken either in surgery or endoscopy. Histopathology of the samples returned diagnosis of jejunitis, gastric serositis, necrotizing duodenitis, eosinophilic colitis, and Helicobacter infection.

Five of the cases had concurrent disease. These diseases included cystitis, eosinophilic bronchitis, salmonella infection, vascular ring anomaly with resulting megaesophagus, myasthenia gravis with resulting megaesophagus, and splenic enlargement.

In 16 of the 17 cases, an exploratory laparotomy was performed along with gastric derotation (when indicated) and gastropexy. One case was euthanized prior to surgical intervention (Frendin et al., 1988). In 5 of the 16 surgical cases, the stomach was described as being in a normal position with no abnormality in position. The stomach in each case was flaccid and easily displaced manually, which explains the intermittence of presenting clinical signs.

Fourteen of the 16 surgical cases had dramatic improvement of clinical signs after surgical intervention. One case died postoperatively due to aspiration pneumonia (Paris et al., 2011), one was euthanized 3 weeks postoperatively due to an unrelated problem (Frendin et al., 1988), and one was euthanized 8 months postoperatively due to acute heart disease (severe biventricular cardiac dilatation) (Frendin et al., 1988). Boothe and Ackerman (1976) describe a case that died 7 months after gastropexy due to gastric torsion.

The presented case in this manuscript was consistent with the presenting signs, breed, and age that is common with CGI. Initial radiographs revealed a compartmentalized but normal sized stomach, which is consistent with previous reports. This case was unique in that the dynamic nature of CGI was documented using computed tomography (CT). In sternal recumbency, the stomach was malpositioned with the pylorus to the left of the fundus. When the patient was repositioned in dorsal recumbency, normal gastric anatomy was appreciated. This further highlights the dynamic nature of gastric positioning in CGI cases, and demonstrates the challenge of obtaining a CGI diagnosis. This case also further supports the need for multiple diagnostic modalities to demonstrate the abnormal stomach orientation that is required to diagnose CGI.

In the presented case, CGI progressed to gastric dilatation with volvulus, which emphasizes the 
continuum that GDV and CGI share. This case explains the potential life-saving benefit of early diagnosis.

Abdominal celiotomy with gastropexy is considered the therapy of choice in CGI cases, and the benefits of gastropexy as a prophylactic to prevent CGI from evolving to GDV are widely accepted. However, exploratory celiotomy with gastropexy was declined in the presented case due to concurrent intrathoracic disease. It must be understood that gastropexy procedures frequently move the pyloric antrum into a non-anatomic location, which can lead to signs of restrictive pyloric disease (Sutton et al., 2015; Tomlinson et al., 2016). CGI disease must be on the differential list of any dog with chronic intermittent vomiting, unexplained weight loss, mild chronic bloating, borborygmi, and eructation (Leib et al., 1987). Two of the cases in literature describe megaesophagus as a comorbidity. Altered GI motility may be a contributing factor in the development of CGI.

The dynamic nature of CGI and frequently normal radiographic anatomy suggests that no single diagnostic modality should be relied on to make a definitive diagnosis of CGI. This author recommends several radiographic views in any suspected case (Frendin et al., 1988; Paris et al., 2011). These include standard lateral and dorsoventral or ventrodorsal views as well as oblique views of the abdomen to help demonstrate the intermittent malpositioning of the aboral stomach. It should also be understood that radiography may not be conclusive and that other imaging such as contrast radiography, abdominal ultrasound, and CT may be needed. In several of the previously reported cases, the stomach was in a normal anatomic position on abdominal celiotomy but was easily displaced manually. Exploratory celiotomy should be considered in any case with presenting signs consistent with CGI with normal diagnostic imaging. In conclusion, CGI is a challenging diagnosis to make, and several diagnostic modalities may be required, including standard radiography, abdominal ultrasound, contrast studies, endoscopy, and CT. Several radiographic views are recommended in suspect CGI cases including standard lateral and ventrodorsal views. However, dorsoventral and oblique views may be necessary to help demonstrate gastric malpositioning. It must be understood that gastrointestinal orientation may change between views, and this finding should heighten the index of suspicion for CGI. Abdominal laparotomy should be considered in each case to determine severity of affected gastric tissue, to explore and address any devitalized tissue, and to perform a prophylactic gastropexy in an appropriate location.

\section{Acknowledgements}

The authors would like to thank Oklahoma State University College of Veterinary Health Sciences for their support of this work.

\section{Conflict of interest}

The Authors declare that there is no conflict of interest.

\section{References}

Boothe, H.W. and Ackerman, N. 1976. Partial gastric torsion in two dogs. J. Am. Anim. Hosp. Assoc. 12, 27-30.

Burrows, C., Bright, R. and Spencer, C. 1985. Influence of dietary composition on gastric emptying and motility in dogs: potential involvement in acute gastric dilatation. Am. J. Vet. Res. 46, 2609-2612.

Evans, K. and Adams, V. 2010. Mortality and morbidity due to gastric dilatation-volvulus syndrome in pedigree dogs in the UK. J. Small Anim. Pract. 51, 376-381.

Fossum, T.W. 2007. Small Animal Surgery. $3^{\text {rd }}$ Ed. Elsevier Health Sciences.

Frendin, J., Funkquist, B. and Stavenborn, M. 1988. Gastric displacement in dogs without clinical signs of acute dilatation. J Small Anim Pract. 29, 775779.

Funkquist, B. 1969. Gastric torsion in the dog. Nonsurgical reposition. J. Small Anim. Pract.10, 507511.

Funkquist, B. 1979. Gastric torsion in the dog-I. Radiological picture during nonsurgical treatment related to the pathological anatomy and to further clinical course. J. Small Anim. Pract. 20, 73-91.

Gazzola, K. and Nelson, L. 2014. The Relationship Between Gastrointestinal Motility and Gastric Dilatation-Volvulus in Dogs. Top. Companion Anim. Med. 29(3), 64-66.

Glickman, L.T., Glickman, N.W., Pérez, C.M., Schellenberg, D.B. and Lantz, G.C. 1994. Analysis of risk factors for gastric dilatation and dilatation volvulus in dogs. J. Am. Vet. Med. Assoc. 204, 1465-1471.

Glickman, L.T., Glickman, N.W., Schellenberg, D.B., Simpson, K. and Lantz, G.C. 1997. Multiple risk factors for gastric dilatation-volvulus syndrome in dogs: a practitioner/owner case-control study. J. Am. Anim. Hosp. Assoc. 33, 197-204.

Glickman, L.T., Glickman, N.W., Schellenberg, D.B., Raghavan, M. and Lee, T.L. 2000a. Incidence of and breed-related risk factors for gastric dilatationvolvulus in dogs. J. Am. Vet. Med. Assoc. 216, 4045.

Glickman, L.T., Glickman, N.W., Schellenberg, D.B., Raghavan, M. and Lee, T. 2000b. Non-dietary risk factors for gastric dilatation-volvulus in large and giant breed dogs. J. Am. Vet. Med. Assoc. 217, 1492-1499.

Leib, M. and Blass, C. 1984. Acute Gastric Dilatation in the Dog: Various Clinical Presentations. The Compendium Cont. Edu. 6, 707-713.

Leib, M., Monroe, W. and Martin, R. 1987. Suspected chronic gastric volvulus in a dog with normal 
gastric emptying of liquids. J. Am. Vet. Med. Assoc. 191, 699-700.

Matthiesen, D. 1983. The gastric dilatation-volvulus complex: medical and surgical considerations. J. Am. Anim. Hosp. Assoc. 19, 925-932.

Paris, J.K., Yool, D.A., Reed, N., Ridyard, A.E., Chandler, M.L. and Simpson, J.W. 2011. Chronic gastric instability and presumed incomplete volvulus in dogs. J. Small Anim. Pract. 52, 651655.

Sutton, J.S., Steffey, M.A., Bonadio, C.M. and Marks, S.L. 2015. Gastric malpositioning and chronic, intermittent vomiting following prophylactic gastropexy in a 20-month-old great Dane dog. Can. Vet. J. 10, 1053-1056.

Theyse, L., Van de Brom, W. and Van Sluijs, F. 1998.
Small size of food particles and age as risk factors for gastric dilatation volvulus in Great Danes. Vet. Rec. 143, 48-50.

Tobias, K.M. and Johnston, S.A. 2012. Veterinary Surgery: Small Animal. Saunders Elsevier.

Tomlinson, A.W., Lillis, S.M., German, A.J. and Burrow, R.D. 2016. Pyloric localisation in 57 dogs of breeds susceptible to gastric dilatation-volvulus using computed tomography. Vet. Rec. 179(24), 626.

Wingfield, W.E., Cornelius, L.M. and Deyoung, D.W. 1974. Pathophysiology of the gastric dilatationtorsion complex in the dog. J. Small Anim. Pract. $15,735-739$. 